\title{
DEVELOPING A REAL-TIME (LABVIEW BASED) PRODUCTIVITY MEASUREMENT SYSTEM FOR TEXTILE FACTORIES
}

\author{
Ihab El-Sayed ${ }^{\text {, Mohamed Zahrant }}{ }^{2,3}$, Yousry Atia ${ }^{2,4}$ \\ 1. Jazan University, Faculty of Engineering, Industriai Engineering Department, KSA: \\ dr_ihab2010@yahoo.com \\ 2. Electronics Research Institute, PV Dept., NRC Blg., El-Tahrir St., Dokki, 12311-Giza, Egypt \& \\ 3. Jazan University, Faculty of Engineering, Electrical Engineering Department, KSA: \\ mbazahran_2007@yahoo.com \\ 4. Jazan Techrical College, Electrical Engineering Department, KSA : yousry_atia@yahoo.com
}

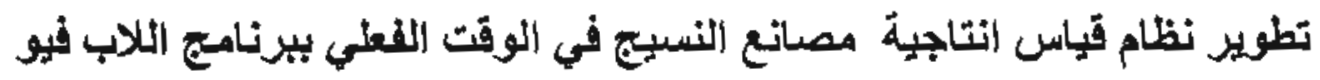

خلإهنة

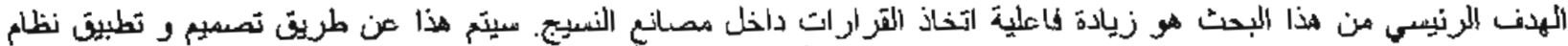

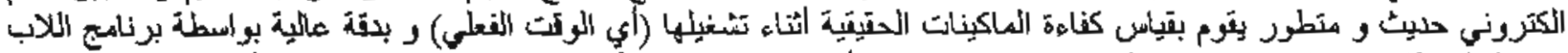

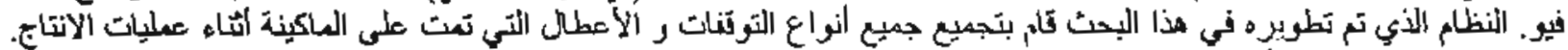

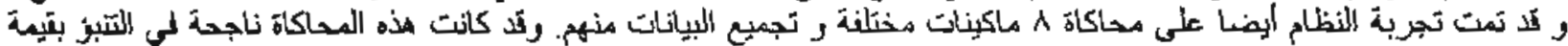

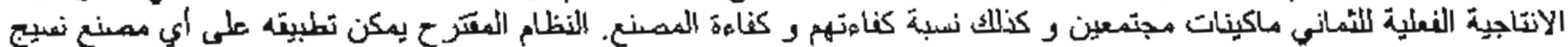

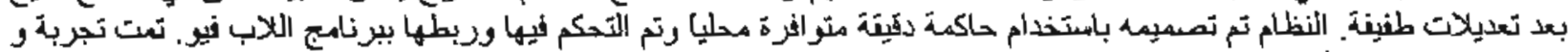

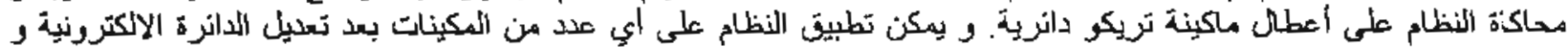
يرنانعج النُككم.

\section{ABSTRACT}

The main goal of this paper is to increase decision effectiveness in textile manufacturing through the design and implementation of a new real-time (on-line) LabView based software and hardware system for measuring actual and accurate total stoppage time of the machines in the entire factory. The data would be utilized in predicting the total productivity of any Textile manufacturing organization. The system was designed using a microcontroller and LabView 8.5 from National Instruments. It was simulated on a circular knitted machine. The system could be applied on any number of machines after slight modification in the electronic circuits and the software.

Key words: Textile manufacturing, Labview VI, Microcontroller, Productivity, Efficiency

\section{INTRODUCTION}

Computerized monitoring in any textile and knitting factory (TKF) is becoming one of the very essential issues these days. Factories are in need to know their process capability, productivity and their efficiency.
Many system has been designed to monitor the productivity, operability and overall efficiency and capability. Factory managers need to know the current situation of the current order, they need to determine when the order will be finished and what time the production

Accepted June 30, 2009. 
supervisor will start in the new order, and when it is expected to be finished.

In many cases Managers need to have remote data access possibilities. Also archiving production data is important as well as production data transfer.

All factories are in vital need to have a reliable production monitoring system, depending or their level.

Starting from a small workshop with one machine up to a huge size enterprises, a flexible and easy to upgrade system must be in hand of any factory owner or production manager in order to cope with his need and growing business opportunity.

Our developed system in this research paper will achieve most of the above mentioned required items.

This includes, reliable production monitoring system, capability of data transfer, capability of archiving production data, on-line production monitoring and management, and finally remote data access.

Our system have a very good advantage, being locally developed and can be used for entry level (very small enterprise) and is upgradable to keep performing with business growing up in (Small and Medium enterprises).

The system can be customized to any textile process. The position of the sensors responsible for sensing the status of the machine would be modified according to the relevant textile process in hand. Some modification are also required in the math script of the LabView (Virtual Instruments) VI.

Many similar systems are available in this field. [1-5] Research Innovation Textile Machinery (RITM) has reported in [1] that, RTM has always been an innovative partner for the yam production factory management.

Accutrack Automation has reported in [3] that, there are many benefits of a real time measurement collection and process monitoring system (Figure 1). Some of these benefits are:-

\section{Reduced Downtime}

Stopped machine are highlighted on the factory floor plan. The current and past reason for stoppage is logged to the system database to detailed analysis. It is therefore easy to determine and correct problems causing machine downtime.

\section{Measurement of machine and Plant efficiency providing reference or targets}

All efficiency of all machines is calculated in real time. This data is logged as a line graph for reference at any time. The machine efficiencies are also summed to generate a plant efficiency figure which is also logged to the plant efficiency line graph. This provides a reference against which future efficiencies can be measured and improved upon.

\section{Increased Efficiency through accurate information}

Accurate information in real times means accurate decisions can be made more quickly. Having fact and justification to make decision is crucial in any environment.

\section{Real time machine speed monitoring}

Machine running at slow speed beyond normal warm times can be measured, monitored and recorded. Also, machine running below manufacturer's specification can also be measured. Both these result in loss of production

\section{Real time job tracking}

Once a job is scheduled in to the system it can be tracked in real time. The progress of the job, its predicted completion time is calculated in real time and compared against targets. Any jobs that are behind schedule and late are automatically highlighted. This 
enables better planning and customer relations as they can be informed quickly.

\section{Operator management}

Operator working patterns and shift setup can be entered into the system. Operators are automatically logged in and out of the system and assigned to relevant machine during the shifts. With this setup it is possible to monitor operator performance and improve efficiency.

\section{Quality Reports}

Quality and accurate reports can be generated at any time to view current status or machines or production. Also, an end of shift report can be automatically generated summarising the efficiency and productivity of each machine during the shift. This provides vital data to enable patterns to be recognised such as poor night shift efficiency.

\section{DISTRIBUTED MEASUREMENT AND TRACKING DATA OVER INTRANET AND INTERNET}

Often many departments require measurement data and information on production and machines. This real time data can be viewed anywhere using software which provides this valuable facility.

Aiwina Heng et.al, have reported in [6] that, the ability to forecast machinery failure is vital to reducing maintenance costs, operation downtime and safety hazards. Recent advances in condition monitoring technologies have given rise to a number of prognostic models that attempt to forecast machinery health based on condition data.

Maintenance cost effectiveness; asset availability and operational safety have a direct impact on the competitiveness of organizations. To comply with this requirement, condition-based maintenance (CBM) has become established throughout industry. CBM is a philosophy of maintaining engineering assets based on nonintrusive measurements of their condition as well as on maintenance logistics. Most of the existing condition monitoring (CM) systems focuses on the acquisition of asset condition information and the detection/classification of faults based on the acquired data. These technologies enable an existing problem to be detected, diagnosed and corrected before breakdowns or other serious consequences occur. However, the related and yet more important question is how to utilize this asset health information for predicting remaining as set lifetime, optimizing maintenance schedules and ultimately maximizing organizational efficiency. Enhanced application of CBM is thus through prognosis-the forecast of an asset's remaining operational life, future condition, or risk to complete operation. Several valuable models have been proposed in the researcb area of machinery fault prognosis [6].

Ufuk Cebeci has reported in [7] that, an enterprise resource planning system (ERP) is the infomation backbone of a company that integrates and automates all business operations. It is a critical issue to select the suitable ERP system which meets all the business strategies and the goals of the company. ERP systems are becoming more necessary for almost every firm to improve the competitiveness. According to the success of the implementation of ERP system; companies can obtain a competitive advantage in the global market rapidly.

Chance Elliott et. $\mathrm{Al}$, have reported in [10] that, National Instruments LabVIEW is a graphical programming language that has its roots in automation control and data acquisition. Its graphical representation, similar to a process flow diagram, was created to provide an intuitive programming environment for scientists and engineers. The language has matured over the last 20 years to become a general purpose programming environment. LabVIEW has several key features which make it a good choice in an 
automation environment. These include simple network communication, turnkey implementation of common communication protocols (RS232, etc.), powerful toolsets for process control and data fitting, fast and easy user interface construction, and an efficient code execution environment. We discuss the merits of the language and provide an example application suite written in-house which is used in integrating and controlling automation platforms.

\section{MONITORING AND MANAGEMENT OF TKF PRODUCTION}

Our proposed system was initially designed to monitor any textile process and help in predicting its efficiency. This would be done based on a real time data acquisition system.. This system has an important advantage being reliable, accurate and representing actual data. It will be applied in this paper on a circular knitting machine.

There are many other data which are of interest to textile process managers on a daily bases.

These includes Date, Time, File name, Computer name, Computer Location, Machine Type, Model, Gauge, Machine Number, date of starting knitting job as well as its accomplishing time.

The proposed system will be capable of the following:

1. Individual machines efficiency calculations

2. Sum, Average and Range of Individual machines efficiency calculations

3. Calculation of Factory efficiency from the above.

4. Logging the current and past reason of stopping for better cause analysis and determining.

5. Machine speed measuring and average during production
6. Logging progress of the order for better planning.

7. Operator log- in and operator assigning of relevant machines and operator performance monitoring.

8. Producing Quality report, data like time of start to end of knitting job, type of fault, their frequencies.

The proposed system is capable of Data Logging, Data transferring, possible interfacing with (Enterprises Resource Planning ERP) and or (Manufacturing Resource Planning MRP) systems

Possibility of Connecting data via internet and SMS (GSM Modem) for reporting purposes is also available. It also makes Real Time data of the factory available for viewing anywhere anytime in the world.

\section{MODELING AND SIMULATION OF THE MONITORING SYSTEM FOR TEXTILE}

The monitoring system for textile machine $\mathrm{MV}$ 4-3.2 II from Mayer and Cie type is built up using an advanced software program; LabVIEW. The block diagram is shown in Figure 3. The machine operation signals are acquired using an implemented data acquisition system based on microcontroller and $\mathrm{PC}$ serial port interfacing. The proposed acquisition system is reliable, cheap and has reconfigured capability. The block diagram shows the serial interface initialization in LabVIEW serial visa. The acquired data are controlled by the given word in the write buffer. This word is received and analyzed in the microcontroller embedded program to start the data acquisition with relevant sampling time. An analog multiplexing circuit is added to acquire a huge number of analog variables needed to monitor the health state of Textile machine. The analog signals are scanned and the converted data are stored in a specific memory location in microcontroller flash memory. The serial interface has a routine program to transfer this data to $\mathrm{PC}$ for the 
purpose of textile machine process control. An array of logical signals (true or false) is collected from the distributed sensors on the textile machine to follow up the discrete status of control limit switches.

A data processing stage is included in the LabVIEW program block diagram. This stage is responsible for dissipation the stream of data based on the index of each analog variable. Then the sorted data could be used as an input signal for the math-script module in the LabVIEW. In this module, the logical monitoring and the mathematical function are designed and implemented.

The monitoring system user interface is shown in the front panel of developed LabVIEW program shown in Figure 4. In this figure a monitoring menu for eight textile machines is given. Each machine is represented by two analog values; one for its operability (OP- $x$ ) and the other for its productivity (PR-x). One image for textile machine $\mathrm{MV}$ 4-3.2 II from Mayer and Cie type is inserted it the front panel to show where each sensor is locatud. The other values stated in the front panel are dedicated for the control parameters of serial interface with the data acquisition system (microcontroller with its peripherals) and for the sampling time, time elapsed, etc...

\section{ANALOG AND DIGITAL SIGNALS DESCRIPTION}

Calculation of Time Consumed in Maintaining the Machine in Productive Condition

\section{MEASUREMENT OF PRODUCTIVTTY}

The main objectives of Productivity Measurement are:

To Study the Performance of a System

To have relative comparison of different systems for a given value of input.
To compare the actual Productivity with the planned Productivity

\section{Total Productivity}

Many reasons during the process could affect the total productivity percentage. This include all time losses due to repairing and knotting of broken yarn, changing empty cones, replacing broken needle, running in low speed after knotting a broken yam, cutting fabric rolled on the take down mechanism, attaching fabric end firmly on the take down mechanism, cleaning the machine, oiling the machine and any other maintenance routines.

From Table (1)Total Losses could be calculated according to the following equation:

Total Losses $=\Sigma\left(T_{c c}+T_{c b n}+T_{\text {idcf }}+T_{\text {ffeff }}+T_{\text {bby }}+\right.$ $\left.T_{s s}+T_{p s}+T_{\text {others }}\right) \ldots \ldots \ldots \ldots \ldots . . .(1)$

The above are description for most of the expected losses time which could be analyzed and hence minimized. It is envisaged that these would help in increase decision effectiveness in knitting manufacturing unit, and hence, applied to other manufacturing processes. All managers in all levels need to be capable of taking effective decision.

By taking the summation of the total time losses as in equation 1, into consideration, Managers at all levels could predict their job efficiency, machine efficiency, operator efficiency and over all factory efficiency. Our proposed system will facilitate all these calculations..

Productivity management is one of the important functions of industrial engineering departments in companies. Productivity measurement facilitates planning and controlling productivity levels in the companies.

The Textile machine monitoring signals can be listed as shown in the following items. A brief description will be added under each one.

OPERABULTY, 
1. On/off monitoring, it monitors the numbers of stoppages when happened and used to initialize counter to count number of stops and timer for elapsed time for each stop. The elapsed times are accumulated to estimate the total elapsed time during whole the shift. This will be used in machine efficiency calculation; this signal comes out from the machine motor power supply sensor, PS (power supply) sensor, CBN (changing broken needle) and CBY (changing broken yam) see Figure 4.

2. Operation speed, it monitors the speed value of the machine which could be found in one of two cases; high speed (normal) and low speed (after maintenance or operator interaction). This will be used in machine efficiency calculation, SS (speed sensor) sensor, see Figure 4.

3. Loading period, sometimes called doffing period, it happens frequently twice in the shift, once each 4 hours for normal operation and it consumes about 10 15 minutes each time, $\mathrm{CC}$ (changing cone) sensor, see Figure 4.

4. Removing fabric roll, it depends on the fabric type, for thick and sometimes called coarse fabric, it happens more than four times in the shift while in fine fabric it happens twice per shift and each time it consumes 2-3 minutes, TDCF (take down cutting fabric) sensor, see Figure 4.

5. Oiling the machine, normally the machine oiling processed automatically but sometimes we urgent need to lubricate the machine, this time affects the efficiency of the machine and it will be depicted in the ondoff monitoring and operation speed monitoring also, PS sensor, see Figure 4.

6. Repairing Brocken yam, this case will be depicted also in the on/off monitoring, $\mathrm{CBY}$ sensor, see Figure 4.
7. Changing empty cone, this case will be depicted also in the on/off monitoring, $\mathrm{CC}$ sensor, see Figure 4.

\section{PRODUCTIVITY,}

Time accumulation, it is an analog value depends on many digital sensors and the operation of software counters and times. It calculates the absolute time (time from the beginning of shift till the end or time from the beginning the order production till the end) and stoppage times (accumulated times of stoppage happened because of any one of the previous described items in operability section),

\section{Measurement of Productivity}

The main objectives of Productivity Measurement are:

1- To Study the Performance of a System.

2- To have relative comparison of different systems for a given value of input.

3- To compare the actual Productivity with the planned Productivity.

\section{EFFICIENCY}

The machine efficiency is calculated based on its operability and productivity, it depends on the sensors and signals which described in the operability section, finally it could be calculated by the following equation:

of $f(\%)=\frac{\text { (net oporation t(mo) }}{\text { absolut o time }} \cdot 100$

o/f (\%) $=\frac{\text { (ebsolute tume - accumulated stoppage tumos) }}{\text { absolute time }} \times 100$

Where:

Accumulated stoppage times $=$ Total Losses from equation (1).

In the math-script module, the digital signals, analog values of the machine variables are used as input data, then the mathernatical equation for calculating efficiency is applied. The software counters and times are built in 
the math-script module by the help of logical expressions.

\section{EFFICIENCY CALCULATIONS}

1 day $=3$ Shifts, I Shift $=8$ hours

Circular Knitting Machine Production / Shift, Machine Type and Model, Circular Knitting

From equation (1), all losses will be collected on-line from the microcontroller.

Total Losses $/$ Shift $/$ Machine $=\Sigma T_{\text {Losses }}$ (in minutes)

Machine Efficiency $=\dot{\eta}_{\text {Tr/c }}(\%)$

$\dot{\eta}_{m / c}=100 *\left(480-\Sigma T_{\text {Losses }}\right) / 480$

Total Factory Efficiency $=\Sigma \dot{n}_{\text {mic }}$

\section{Conclusion}

The proposed system was capable of logging and recording all type of time losses during the production process. It was logging on-line (real-time) accurate times. The system was simulated on multiple machines ( 8 in the case of this paper). It is possible to apply it on virtually any number of machines according to the manufacturing unit available. The Efficiency of each machine was displayed on the Front Panel of the LabView Virtual Instruments (VD) and next to them the average Efficiency (Total Manufacturing Unit) was also displayed for managerial decisions ,. See figure 4. The signals and the entire data could be delivered to any ERP or MRP system installed in the manufacturing unit.

The system could be programmed to count the number of yan breakage in each machine and decide whether or not to continue with this yam lot as per certain pre-determined value.

\section{References}

[1] Research Innovation Textile Machinery (RITM), allée Charles Baron Z.I. Les Auréats FR-26014 Valence Cedex, T: +33 (0)475752600 F: +33 (0)475752601 E: sales@ritm-fr.com, www.ritm-fr.com or www.textile-machinery.info.

[2] F. CusD, M. Milfelner, J. Balic, "An intelligent system for monitoring and optimization of ball-end milling process", Jounal of Materials Processing Technology 175 (2006) 90-97

[3] ACCUTRACK AUTOMATION LTD, "On-Machine Measurement, Project Handbook Supplement Production Measurement \& Monitoring System, For Circular Knitting Machine", 44 OVERTON ROAD, LEICESTER, LE5 OJA, ENGLAND, TEL; +44 1162215545 , FAX: +44 116 2215546, Website: www.accutrack.co.uk

[4] Dr. 1. Antoniadis - Rodax Corporation S.A. Industry, "Using LabVIEW and PXI for an Online Mechanical Parameters Monitoring System for a Diesel Generator Unit Set", National Technical University of Athens Control Systems Section, Tel: +30210772 1524, Fax: +30 210772 1525, E-mail: antogian@central.ntua.gr,

[5] Texion, "Textile Technology",

[6] Aiwina Heng, AndyC.C.Tan, Joseph Mathewa, Neil Montgomery, Dragan Banjevic and AndrewK.S.Jardine, "Intelligent condition-based prediction of machinery reliability", Expert Systems with Applications 36 (2009) 8839-8848

[7] Ufuk Cebeci, "Fuzzy AHP-based decision support system for selecting ERP systems in textile industry by using balanced scorecard", Expert Systems with Applications 36 (2009) 8900-8909

[8] Jayanta Deb Mondol a,*, Yigzaw G Yohanis a, Brian Norton b, "Optimising the economic viability of grid-connected 
photovoltaic systems", Applied Energy 86 (2009) 985-999.

[9] Leyla Ozgener $a *$, Onder Ozgener b, 'Monitoring of energy exergy efficiencies and exergoeconomic parameters of geothermal district heating systems (GDHSs)", Applied Energy 86 (2009). 1704-1711.

Table 1, different time losses reasons and their symbols

\begin{tabular}{|c|c|}
\hline Time & Symbol \\
\hline Changing Cones & $\mathrm{T}_{c c}$ \\
\hline Changing Broken Needle & $\mathrm{T}_{\mathrm{cbn}}$ \\
\hline Take Down Cutting Fabric & $\mathrm{T}_{\mathrm{cdcf}}$ \\
\hline Fixing Fabric End to Fabric Roller & $\mathrm{T}_{\mathrm{ffefr}}$ \\
\hline Knotting a Broken Yarn & $\mathrm{T}_{\mathrm{kby}}$ \\
\hline Machine Speed Low or High & $\mathrm{T}_{\mathrm{ss}}$ \\
\hline Machine Power Supply & $\mathrm{T}_{p s}$ \\
\cline { 1 - 1 } Oiling the Machine (at zero speed) & \multirow{2}{*}{$\mathrm{T}_{\mathrm{others}}$} \\
\cline { 1 - 1 } Rotating Machine on Low Speed & \\
\cline { 1 - 1 } Cleaning Machine (at zero speed) & \\
\cline { 1 - 2 }
\end{tabular}

[10] Chance Elliott, Vipin Vijayakumar, Wesley Zink, and Richard Hansen, 'National Instruments LabVIEW: A Programming Environment for Laboratory Automation and Measurement", JALA February 2007

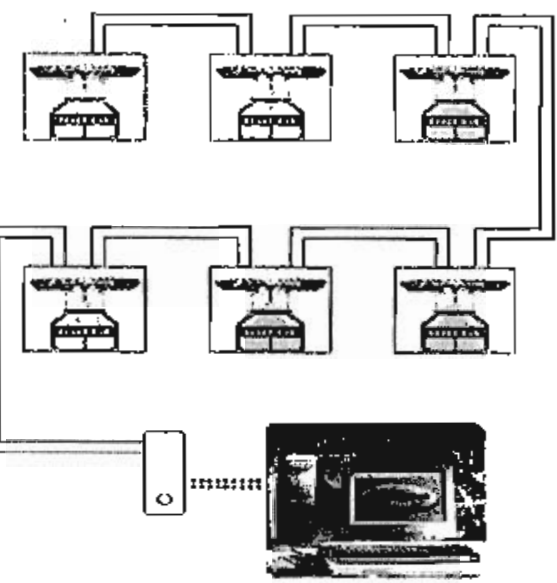

Figure 1, Accutrack Automation Ltd. monitoring system block diagram

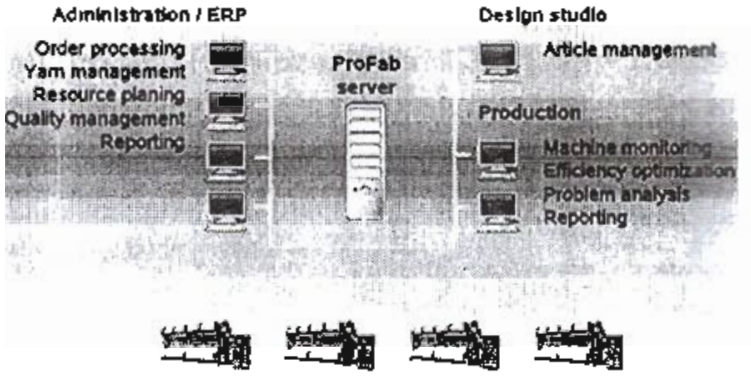

Figure 2, ProFab topology [5] 


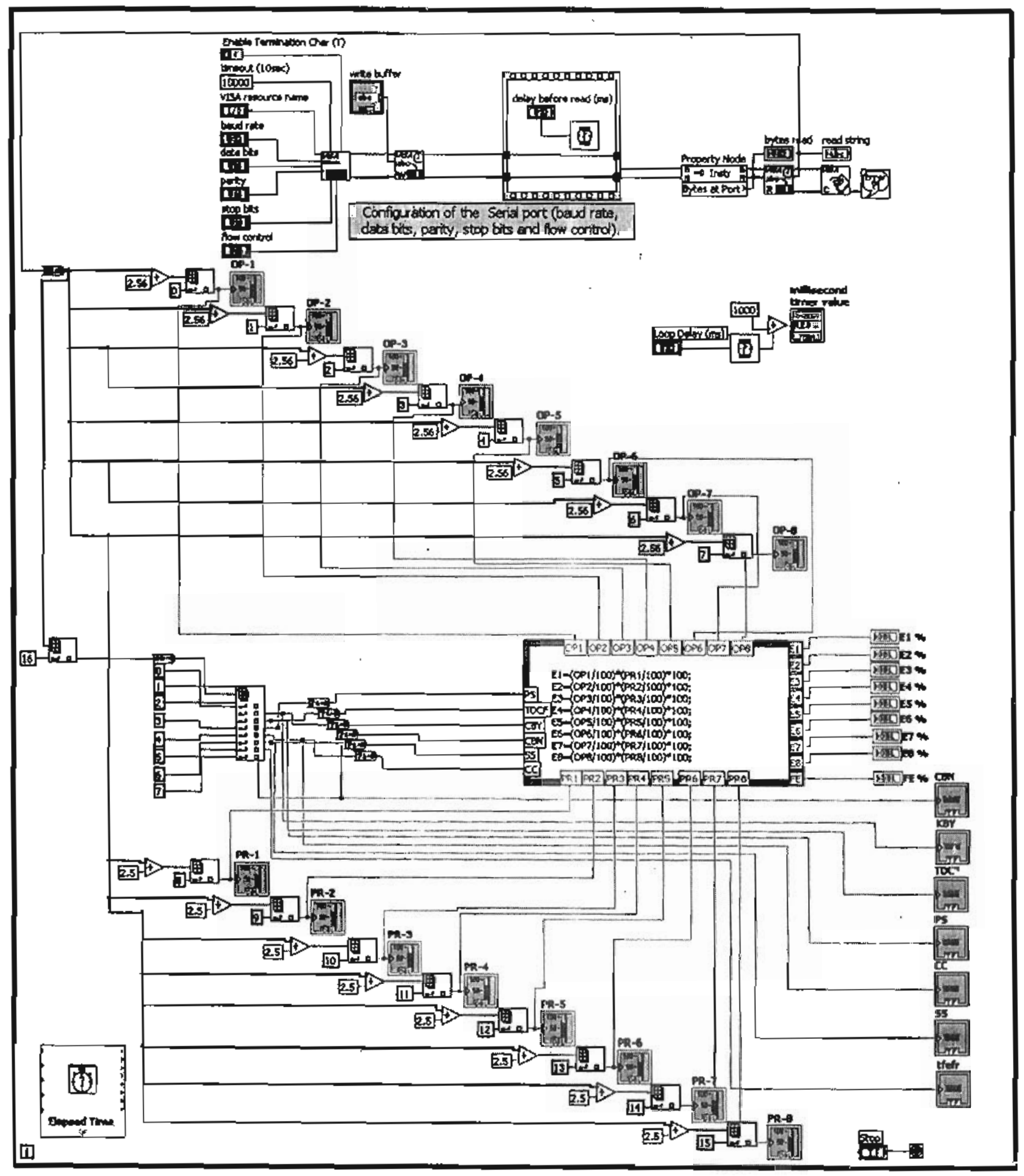

Figure 3, block diagram of textile monitoring system. 


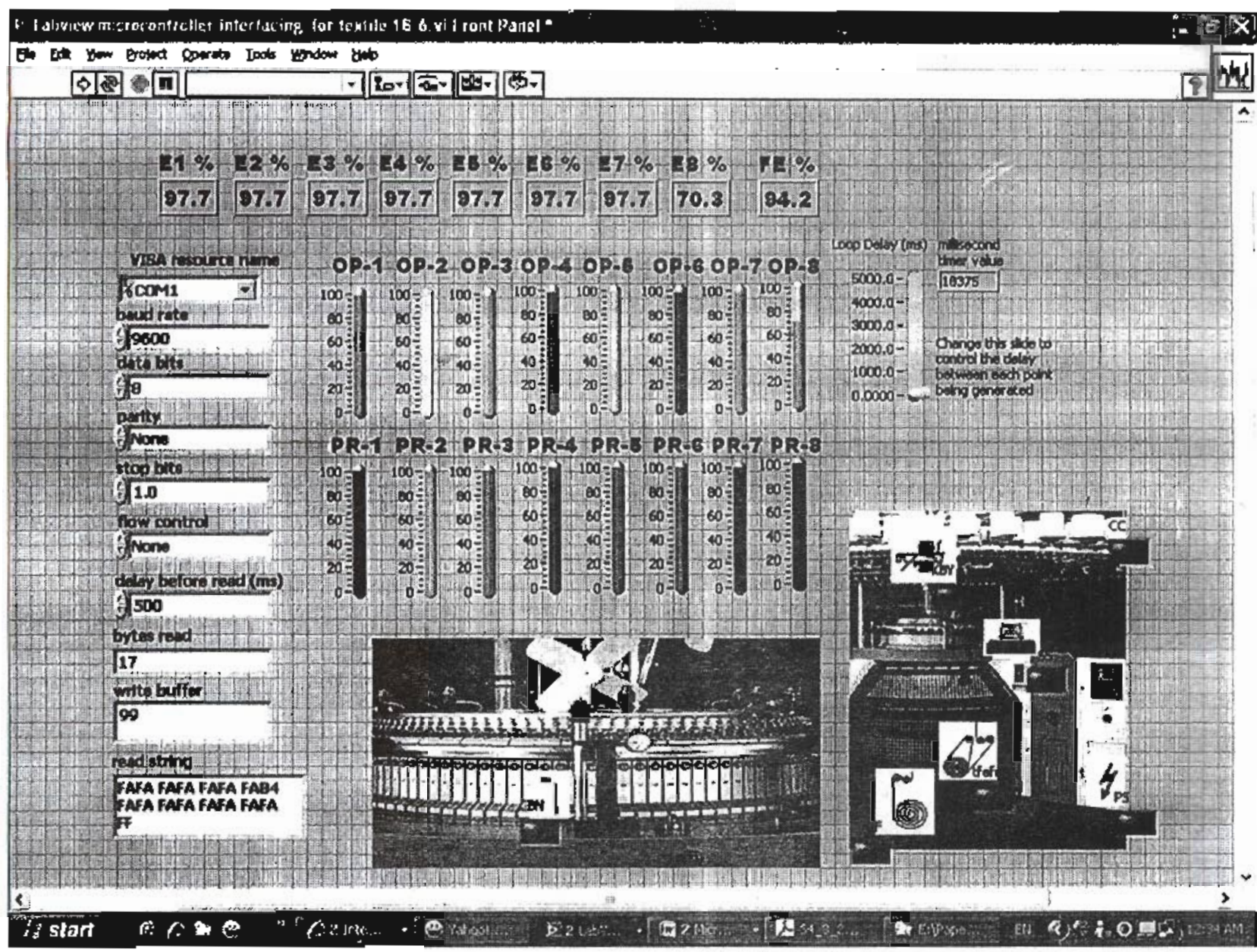

Figure 4, front panel of eight textile machines monitoring system 